\title{
TITLE:
}

\section{Angular momentum at null infinity in five dimensions}

\section{$\operatorname{AUTHOR}(S)$ :}

Tanabe, Kentaro; Tanahashi, Norihiro; Shiromizu, Tetsuya

\section{CITATION:}

Tanabe, Kentaro ... [et al]. Angular momentum at null infinity in five dimensions. Journal of Mathematical Physics 2011, 52(3): 032501.

ISSUE DATE:

2011-03

URL:

http://hdl.handle.net/2433/140402

RIGHT:

(C) 2011 American Institute of Physics. 


\title{
Angular momentum at null infinity in five dimensions
}

\author{
Kentaro Tanabe, ${ }^{1, a)}$ Norihiro Tanahashi, ${ }^{2, b)}$ and Tetsuya Shiromizu ${ }^{3, c)}$ \\ ${ }^{1}$ Yukawa Institute for Theoretical Physics, Kyoto University, Kyoto 606-8502, Japan \\ ${ }^{2}$ Department of Physics, University of California, Davis, California 95616, USA \\ ${ }^{3}$ Department of Physics, Kyoto University, Kyoto 606-8502, Japan
}

(Received 13 October 2010; accepted 5 February 2011; published online 3 March 2011)

\begin{abstract}
In this paper, using the Bondi coordinates, we discuss the angular momentum at null infinity in five dimensions and address the Poincare covariance of the Bondi mass and angular momentum. We also show the angular momentum loss/gain law due to gravitational waves. In four dimensions, the angular momentum at null infinity has the supertranslational ambiguity and then it is known that we cannot construct welldefined angular momentum there. On the other hand, we would stress that we can define angular momentum at null infinity without any ambiguity in higher dimensions. This is because of the nonexistence of supertranslations in higher dimensions. ( 2011 American Institute of Physics. [doi:10.1063/1.3559917]
\end{abstract}

\section{INTRODUCTION}

Inspired by the recent progress of the string theory, the importance of the gravity theory in higher dimensional space-times is steadily growing. However, there are still many remaining issues to be investigated in higher dimensions. One issue among them is the asymptotic structure. For asymptotically flat space-times, the asymptotic structure is defined at spatial and null infinities. The asymptotic structure at spatial infinity (spi) is well defined by conformal embedding in four ${ }^{1-3}$ and higher dimensions. ${ }^{4}$ The asymptotic structure at null infinity in four dimensions is well studied by many authors. ${ }^{2,-12}$ On the other hand, there are only a few works about the asymptotic structure at null infinity in higher dimensions. Indeed, asymptotic flatness has been defined by using conformal completion method in only even dimensions ${ }^{13-15}$ and by using the Bondi coordinates in five dimensions. ${ }^{16}$

In four dimensions, asymptotic structure at null infinity is often studied by using the conformal embedding. ${ }^{8}$ In this method, we introduce the conformal factor $\Omega \sim 1 / r$ and use $\Omega$ as a coordinate near null infinity $\Omega=0$. In four dimensions, this method provided us successful results for the analysis of asymptotic structure. However, it is turned out that this method does not work well in higher dimensions. In fact, we cannot guarantee the smoothness of gravitational fields at null infinity in the coordinate of $\Omega$, particularly in odd dimensions. ${ }^{13-15,17}$ This is because gravitational wave behaves $1 / r^{(D-2) / 2}$ near null infinity in $D$ dimensions. This means that the conformal completion method might not be the best way to study the issue. Then we must solve the Einstein equations directly to study how the gravitational field is expanded near null infinity in odd dimensions. By introducing the Bondi coordinates instead, we can study the asymptotic structure at null infinity even in odd dimensions without assuming the smoothness of the gravitational fields. Indeed, in this way, we can derive the Bondi mass loss law by gravitational waves in five dimensions. We can also show that the regularity of gravitational field at null infinity and that asymptotic symmetry is the Poincare group, i.e., there are no supertranslations at null infinity in five dimensions ${ }^{16}$ while there are always supertranslations in four dimensions.

\footnotetext{
a)Electronic mail: tanabe @yukawa.kyoto-u.ac.jp.

b)Electronic mail: tanahashi@ms.physics.ucdavis.edu.

c)Electronic mail: shiromizu@tap.scphys.kyoto-u.ac.jp.
} 
The asymptotic symmetry at null infinity in four dimensions is semidirect product of the Lorentz group and the supertranslational group, which is an infinite dimensional translational group. The presence of supertranslations implies the infinite number of the direction of translation, while the Poincare group has only four directions in four dimensions. Because of this infinite directions of translation, we cannot construct well-defined angular momentum in four dimensions. There are many attempts to define of angular momentum at null infinity in four dimensions, whereas all those definitions are suffered from supertranslational ambiguity. ${ }^{18-24}$ On the other hand, in five dimensions, since asymptotic symmetry is the Poincare group, we can expect that angular momentum at null infinity can be defined without any ambiguities. The purpose of this paper is to discuss angular momentum at null infinity in five dimensions. We will see that angular momentum can be defined well and show the Poincare covariance of the Bondi mass and angular momentum. We also look at the angular momentum loss/gain properties due to gravitational waves.

The rest of this paper is organized as follows. In Sec. II, we review our previous work. ${ }^{16}$ Therein we introduce the Bondi coordinates and solve the Einstein equations near null infinity. In Sec. III, we define the Bondi mass and the Bondi angular momentum and derive the Bondi mass loss law and angular momentum loss/gain law by gravitational waves. In Sec. IV, we show the Poincare covariance of the Bondi mass and angular momentum. In Sec. V, we summarize our paper and discuss the extension to higher dimensions than seven. In the Appendix, for comparison, we will consider the angular momentum at null infinity in four dimensions using the Bondi coordinates. This is because we cannot find references which address angular momentum using the Bondi coordinate. We will show that there is always the supertranslational ambiguity in the angular momentum.

\section{THE BONDI COORDINATES AND THE EINSTEIN EQUATIONS}

In this section we review our previous work of Ref. 16 on the asymptotic structure at null infinity in five dimensions. Therein we used the Bondi coordinates, which will be useful to study the behavior of gravitational fields via solving of the Einstein equation.

\section{A. Bondi coordinates}

In the Bondi coordinates $x^{a}=(u, r, \theta, \phi, \psi)$ the metric can be written as

$$
d s^{2}=-\frac{V e^{B}}{r^{2}} d u^{2}-2 e^{B} d u d r+r^{2} h_{A B}\left(d x^{A}+U^{A} d u\right)\left(d x^{B}+U^{B} d u\right),
$$

where $x^{A}=(\theta, \phi, \psi)$ and

$$
h_{A B}=\left(\begin{array}{ccc}
e^{C_{1}} & \sin \theta \sinh D_{1} & \cos \theta \sinh D_{2} \\
\sin \theta \sinh D_{1} & e^{C_{2}} \sin ^{2} \theta & \sin \theta \cos \theta \sinh D_{3} \\
\cos \theta \sinh D_{2} & \sin \theta \cos \theta \sinh D_{3} & e^{C_{3}} \cos ^{2} \theta
\end{array}\right),
$$

and we adopted the gauge condition satisfying $\operatorname{det} h_{A B}=\sin ^{2} \theta \cos ^{2} \theta \cdot u=$ const. are null hypersurfaces and the periods of the coordinates $\theta, \phi$, and $\psi$ are $\pi / 2,2 \pi$, and $2 \pi$, respectively. From the gauge condition, $e^{C_{3}}$ can be written as

$$
e^{C_{3}}=\frac{1+e^{C_{2}} \sinh ^{2} D_{2}+e^{C_{1}} \sinh ^{2} D_{3}-2 \sinh D_{1} \sinh D_{2} \sinh D_{3}}{e^{C_{1}+C_{2}}-\sinh ^{2} D_{1}} .
$$

Then $h_{A B}$ have five functional freedom. In the following we will identify $C_{1}, C_{2}, D_{1}, D_{2}, D_{3}$ as those freedom. In this coordinate system, null infinity is represented by $r=\infty$ and the metric at null infinity is

$$
d s^{2}=-d u^{2}-2 d u d r+r^{2}\left(d \theta^{2}+\sin ^{2} \theta d \phi^{2}+\cos ^{2} \theta d \psi^{2}\right) .
$$




\section{B. The Einstein equations}

To investigate the asymptotic structure at null infinity, we have to solve the Einstein equations near null infinity. Here note that five-dimensional space-times have five degree of freedom of gravitational fields. If we identify $h_{A B}$ as the freedom of gravitational field, $C_{1}, C_{2}, D_{1}, D_{2}, D_{3}$ can be expanded as

$$
\begin{aligned}
& C_{1}\left(u, r, x^{A}\right)=\frac{C_{11}\left(u, x^{A}\right)}{r \sqrt{r}}+\frac{C_{12}\left(u, x^{A}\right)}{r^{2}}+\frac{C_{13}\left(u, x^{A}\right)}{r^{2} \sqrt{r}}+\frac{C_{14}\left(u, x^{A}\right)}{r^{3}}+O\left(r^{-7 / 2}\right), \\
& C_{2}\left(u, r, x^{A}\right)=\frac{C_{21}\left(u, x^{A}\right)}{r \sqrt{r}}+\frac{C_{22}\left(u, x^{A}\right)}{r^{2}}+\frac{C_{23}\left(u, x^{A}\right)}{r^{2} \sqrt{r}}+\frac{C_{24}\left(u, x^{A}\right)}{r^{3}}+O\left(r^{-7 / 2}\right), \\
& D_{1}\left(u, r, x^{A}\right)=\frac{D_{11}\left(u, x^{A}\right)}{r \sqrt{r}}+\frac{D_{12}\left(u, x^{A}\right)}{r^{2}}+\frac{D_{13}\left(u, x^{A}\right)}{r^{2} \sqrt{r}}+\frac{D_{14}\left(u, x^{A}\right)}{r^{3}}+O\left(r^{-7 / 2}\right), \\
& D_{2}\left(u, r, x^{A}\right)=\frac{D_{21}\left(u, x^{A}\right)}{r \sqrt{r}}+\frac{D_{22}\left(u, x^{A}\right)}{r^{2}}+\frac{D_{23}\left(u, x^{A}\right)}{r^{2} \sqrt{r}}+\frac{D_{24}\left(u, x^{A}\right)}{r^{3}}+O\left(r^{-7 / 2}\right), \\
& D_{3}\left(u, r, x^{A}\right)=\frac{D_{31}\left(u, x^{A}\right)}{r \sqrt{r}}+\frac{D_{32}\left(u, x^{A}\right)}{r^{2}}+\frac{D_{33}\left(u, x^{A}\right)}{r^{2} \sqrt{r}}+\frac{D_{34}\left(u, x^{A}\right)}{r^{3}}+O\left(r^{-7 / 2}\right) .
\end{aligned}
$$

The Einstein equations $R_{r r}=0, R_{r A}=0$, and the trace part of $R_{A B}=0$ determine the behavior of $B, U^{A}$, and $V$ near null infinity as

$$
\begin{aligned}
\frac{V}{r^{2}} & =1+\frac{V_{1}\left(u, x^{A}\right)}{r \sqrt{r}}-\frac{m\left(u, x^{A}\right)}{r^{2}}+O\left(r^{-5 / 2}\right), \\
B & =\frac{B_{1}\left(u, x^{A}\right)}{r^{3}}+O\left(r^{-4}\right), \\
U^{A} & =\frac{U_{1}^{A}\left(u, x^{A}\right)}{r^{2} \sqrt{r}}+\frac{U_{2}^{A}\left(u, x^{A}\right)}{r^{3}}+\frac{U_{3}^{A}\left(u, x^{A}\right)}{r^{3} \sqrt{r}}+\frac{U_{4}^{A}\left(u, x^{A}\right)}{r^{4}}+O\left(r^{-9 / 2}\right), \\
h_{A B} & =h_{A B}^{(0)}+\frac{1}{r \sqrt{r}} h_{A B}^{(1)}+\frac{1}{r^{2}} h_{A B}^{(2)}+\frac{1}{r^{2} \sqrt{r}} h_{A B}^{(3)}+O\left(r^{-5 / 2}\right),
\end{aligned}
$$

where

$$
h_{A B}^{(0)}=\left(\begin{array}{ccc}
1 & 0 & 0 \\
0 & \sin ^{2} \theta & 0 \\
0 & 0 & \cos ^{2} \theta
\end{array}\right)
$$

and

$h_{A B}^{(n)}=\left(\begin{array}{ccc}C_{1 n}\left(u, x^{A}\right) & \sin \theta D_{1 n}\left(u, x^{A}\right) & \cos \theta D_{2 n}\left(u, x^{A}\right) \\ \sin \theta D_{1 n}\left(u, x^{A}\right) & C_{2 n}\left(u, x^{A}\right) \sin ^{2} \theta & \sin \theta \cos \theta D_{3 n}\left(u, x^{A}\right) \\ \cos \theta D_{2 n}\left(u, x^{A}\right) & \sin \theta \cos \theta D_{3 n}\left(u, x^{A}\right) & -\left(C_{1 n}\left(u, x^{A}\right)+C_{2 n}\left(u, x^{A}\right)\right) \cos ^{2} \theta\end{array}\right)$,

for $n=1,2,3$. The coefficients in these expansions are all written by the gravitational fields $C_{1}, C_{2}, D_{1}, D_{2}, D_{3}$. From $R_{r r}=0$, we have

$$
B_{1}\left(u, x^{A}\right)=-\frac{1}{8}\left(C_{11}^{2}+C_{11} C_{21}+C_{21}^{2}+D_{11}^{2}+D_{21}^{2}+D_{31}^{2}\right) .
$$


From $R_{r A}=0$,

$$
\begin{aligned}
U_{1}^{\theta} & =\frac{2}{5}\left[\frac{1}{\sin \theta \cos ^{2} \theta} \frac{\partial}{\partial \theta}\left(\sin \theta \cos ^{2} \theta C_{11}\right)+\frac{1}{\sin \theta} \frac{\partial}{\partial \phi} D_{11}+\frac{1}{\cos \theta} \frac{\partial}{\partial \psi} D_{21}-\frac{1}{\sin \theta \cos \theta} C_{21}\right], \\
\sin ^{2} \theta U_{1}^{\phi} & =\frac{2}{5}\left[\frac{1}{\sin \theta \cos \theta} \frac{\partial}{\partial \theta}\left(\sin ^{2} \theta \cos \theta D_{11}\right)+\frac{\partial}{\partial \phi} C_{21}+\tan \theta \frac{\partial}{\partial \psi} D_{31}\right], \\
\cos ^{2} \theta U_{1}^{\psi} & =\frac{2}{5}\left[\frac{1}{\sin \theta \cos \theta} \frac{\partial}{\partial \theta}\left(\sin \theta \cos ^{2} \theta D_{21}\right)+\cot \theta \frac{\partial}{\partial \phi} D_{31}-\frac{\partial}{\partial \psi}\left(C_{11}+C_{21}\right)\right], \\
U_{2}^{\theta} & =\frac{2}{3}\left[\frac{1}{\sin \theta \cos 2 \theta} \frac{\partial}{\partial \theta}\left(\sin \theta \cos ^{2} \theta C_{12}\right)+\frac{1}{\sin \theta} \frac{\partial}{\partial \phi} D_{12}+\frac{1}{\cos \theta} \frac{\partial}{\partial \psi} D_{22}-\frac{1}{\sin \theta \cos \theta} C_{22}\right], \\
\sin ^{2} \theta U_{2}^{\phi} & =\frac{2}{3}\left[\frac{1}{\sin \theta \cos \theta} \frac{\partial}{\partial \theta}\left(\sin { }^{2} \theta \cos ^{2} \theta D_{12}\right)+\frac{\partial}{\partial \phi} C_{22}+\tan \theta \frac{\partial}{\partial \psi} D_{32}\right], \\
\cos ^{2} \theta U_{2}^{\psi} & =\frac{2}{3}\left[\frac{1}{\sin \theta \cos \theta} \frac{\partial}{\partial \theta}\left(\sin \theta \cos ^{2} \theta D_{22}\right)+\cot \theta \frac{\partial}{\partial \phi} D_{32}-\frac{\partial}{\partial \psi}\left(C_{12}+C_{22}\right)\right],
\end{aligned}
$$

and

$$
U_{3}^{\theta}=\frac{10}{7}\left[\frac{1}{\sin \theta \cos ^{2} \theta} \frac{\partial}{\partial \theta}\left(\sin \theta \cos ^{2} \theta C_{13}\right)+\frac{1}{\sin \theta} \frac{\partial}{\partial \phi} D_{13}+\frac{1}{\cos \theta} \frac{\partial}{\partial \psi} D_{23}-\frac{1}{\sin \theta \cos \theta} C_{23}\right],
$$

$$
\sin ^{2} \theta U_{3}^{\phi}=\frac{10}{7}\left[\frac{1}{\sin \theta \cos \theta} \frac{\partial}{\partial \theta}\left(\sin ^{2} \theta \cos \theta D_{13}\right)+\frac{\partial}{\partial \phi} C_{23}+\tan \theta \frac{\partial}{\partial \psi} D_{33}\right]
$$

$\cos ^{2} \theta U_{3}^{\psi}=\frac{10}{7}\left[\frac{1}{\sin \theta \cos \theta} \frac{\partial}{\partial \theta}\left(\sin \theta \cos ^{2} \theta D_{23}\right)+\cot \theta \frac{\partial}{\partial \phi} D_{33}-\frac{\partial}{\partial \psi}\left(C_{13}+C_{23}\right)\right]$.

From the trace part of $R_{A B}=0$, we can obtain

$$
V_{1}\left(u, x^{A}\right)=-\frac{2}{3}\left(\frac{1}{\sin \theta \cos \theta} \frac{\partial}{\partial \theta}\left(\sin \theta \cos \theta U_{1}^{\theta}\right)+\frac{\partial}{\partial \phi} U_{1}^{\phi}+\frac{\partial}{\partial \psi} U_{1}^{\psi}\right) .
$$

These solutions will be needed when we define the Bondi mass and angular momentum in Sec. III and confirm the Poincare covariance of the Bondi mass and angular momenta in Sec. IV.

The equations in the traceless part of $R_{A B}=0$ describe the evolution of $h_{A B}^{(n)}(n>1)$ along the $u$-direction. Since $\partial h_{A B}^{(1)} / \partial u$ does not appear in those equations, we may set it arbitrarily on each $u=$ const. hypersurfaces. This degree of freedom can be regarded as the degree of freedom of gravitational waves. Furthermore, we can see that $h_{A B}^{(2)}$ are time independent $\partial h_{A B}^{(2)} / \partial u=0 .{ }^{16}$ This fact will play a key role when showing the Poincare covariance of the Bondi momentum. The functions $m\left(u, x^{A}\right)$ and $U_{4}^{A}\left(u, x^{A}\right)$ appear as the integration constants of $r$-integration of the equations for each $\left(u, x^{A}\right)$, that is, they are free functions on $u=$ const. hypersurfaces. As seen later, these functions represent the energy and angular momenta contained in $u=$ const. hypersurfaces.

\section{ASYMPTOTIC QUANTITY}

In this section, we define the Bondi mass and angular momenta. The normalization factors are determined so that these quantities coincide to Arnowitt-Deser-Misner (ADM) quantities at spatial infinity. ${ }^{25}$ 


\section{A. The Bondi mass and the Bondi momentum}

We firstly define the Bondi mass and momentum from the asymptotic behaviors of metric components. Since $g_{\text {uи }}$ is expanded near null infinity as

$$
g_{u u}=-1-\frac{V_{1}\left(u, x^{A}\right)}{r \sqrt{r}}+\frac{m\left(u, x^{A}\right)}{r^{2}}+O\left(r^{-5 / 2}\right),
$$

it is natural to define Bondi mass and momentum as

$$
M_{\text {Bondi }}(u)=\frac{3}{16 \pi} \int_{S^{3}} m\left(u, x^{A}\right) d \Omega,
$$

and

$$
P_{\text {Bondi }}^{i}(u)=\frac{3}{16 \pi} \int_{S^{3}} m\left(u, x^{A}\right) \hat{x}^{i} d \Omega,
$$

respectively. In the above $\hat{x}^{i}=(\hat{x}, \hat{y}, \hat{z}, \hat{w})=(\sin \theta \cos \phi, \sin \theta \sin \phi, \cos \theta \cos \psi, \cos \theta \sin \psi)$, which are $l=1$ modes of the scalar harmonics on $S^{3}$ and $d \Omega=\sin \theta \cos \theta d \theta d \phi d \psi$. The Bondi five-momentum $P_{\text {Bondi }}^{a}$ are defined by $P_{\text {Bondi }}^{a}=\left(M_{\text {Bondi }}, P_{\text {Bondi }}^{i}\right)$.

From the Einstein equation of $R_{u u}=0$, we can obtain the Bondi mass loss law such as

$$
\begin{aligned}
& \frac{d}{d u} M_{\text {Bondi }}= \frac{3}{16 \pi} \int_{S^{3}} \frac{\partial m\left(u, x^{A}\right)}{\partial u} d \Omega \\
&=-\frac{1}{16 \pi} \int_{S^{3}}\left\{\left(\frac{\partial C_{11}}{\partial u}\right)^{2}\right.+\frac{\partial C_{11}}{\partial u} \frac{\partial C_{21}}{\partial u}+\left(\frac{\partial C_{21}}{\partial u}\right)^{2}+\left(\frac{\partial D_{11}}{\partial u}\right)^{2}+\left(\frac{\partial D_{21}}{\partial u}\right)^{2} \\
&+\left(\frac{\partial D_{31}}{\partial u}\right)^{2}-\frac{2}{\sin \theta \cos \theta} \frac{\partial}{\partial \theta}\left(\sin \theta \cos \theta \frac{\partial U_{2}^{\theta}}{\partial u}\right) \\
&\left.-2 \frac{\partial^{2}}{\partial \phi \partial u} U_{2}^{\phi}-2 \frac{\partial^{2}}{\partial \psi \partial u} U_{2}^{\psi}\right\} d \Omega \\
&=+\frac{1}{16 \pi} \int_{S^{3}}\left\{\left(\frac{\partial C_{11}}{\partial u}\right)^{2}+\frac{\partial C_{11}}{\partial u} \frac{\partial C_{21}}{\partial u}+\left(\frac{\partial C_{21}}{\partial u}\right)^{2}\right. \\
&\left.+\left(\frac{\partial D_{11}}{\partial u}\right)^{2}+\left(\frac{\partial D_{21}}{\partial u}\right)^{2}+\left(\frac{\partial D_{31}}{\partial u}\right)^{2}\right\} d \Omega \\
& \leq 0 .
\end{aligned}
$$

Thus, it is turned out that the Bondi mass always decreases due to gravitational waves. The total derivative terms in this integral have no contributions to the Bondi mass loss.

We comment on the finiteness of the Bondi mass. In the conformal completion method, we usually define the Bondi mass $M$ at null infinity using Weyl tensor $C_{a b c d}$ as $M \sim \int r C_{u r u r} d S$ with $d S=r^{3} \sin \theta \cos \theta d \theta d \phi d \psi$. Since Eq. (27) implies that $C_{\text {urur }} \sim V_{1} / r^{7 / 2}$ near null infinity, $M$ seems to diverge. Such a singular behavior of the Bondi mass has been pointed out in Ref. 15. However, the solution of Eq. (26) implies that the leading part of the integral $\int r C_{u r u r} d S$ vanishes. That is to say, the finiteness of the Bondi mass is shown by solving the Einstein equations explicitly. Using the solutions of Eqs. (17)-(19) and (26), indeed, we can show the finiteness of the Bondi momentum.

\section{B. The Bondi angular momentum}

Next, let us define the Bondi angular momentum from $u A$ components of the metric. Near null infinity, $g_{u \phi}$ and $g_{u \psi}$ are expanded as

$$
g_{u \phi}=\frac{1}{\sqrt{r}} \sin ^{2} \theta U_{1}^{\phi}+\frac{1}{r} \sin ^{2} \theta U_{2}^{\phi}+\frac{1}{r \sqrt{r}} \sin ^{2} \theta U_{3}^{\phi}+\frac{1}{r^{2}} j^{\phi}+O\left(r^{-5 / 2}\right),
$$




$$
g_{u \psi}=\frac{1}{\sqrt{r}} \cos ^{2} \theta U_{1}^{\psi}+\frac{1}{r} \cos ^{2} \theta U_{2}^{\psi}+\frac{1}{r \sqrt{r}} \cos ^{2} \theta U_{3}^{\psi}+\frac{1}{r^{2}} j^{\psi}+O\left(r^{-5 / 2}\right),
$$

where

$$
\begin{gathered}
j^{\phi}=\sin \theta D_{11} U_{1}^{\theta}+\sin ^{2} \theta C_{21} U_{1}^{\phi}+\sin \theta \cos \theta D_{31} U_{1}^{\psi}+\sin ^{2} \theta U_{4}^{\phi}, \\
j^{\psi}=\cos \theta D_{21} U_{1}^{\theta}+\sin \theta \cos \theta D_{31} U_{1}^{\phi}-\cos ^{2} \theta\left(C_{11}+C_{21}\right) U_{1}^{\psi}+\cos ^{2} \theta U_{4}^{\psi} .
\end{gathered}
$$

Since $U_{1}^{\phi}, U_{1}^{\psi}, U_{2}^{\phi}, U_{2}^{\psi}, U_{3}^{\psi}$, and $U_{3}^{\phi}$ contain only total derivative terms on $S^{3}$ [see Eqs. (17)-(25)], they cannot contribute to the definition of the global quantities. Therefore we define the Bondi angular momenta, $J_{\text {Bondi }}^{\phi}$ and $J_{\text {Bondi }}^{\psi}$, will be naturally defined by

$$
\begin{aligned}
& J_{\text {Bondi }}^{\phi}(u)=-\frac{1}{4 \pi} \int_{S^{3}} j^{\phi} d \Omega, \\
& J_{\text {Bondi }}^{\psi}(u)=-\frac{1}{4 \pi} \int_{S^{3}} j^{\psi} d \Omega .
\end{aligned}
$$

From $R_{u \phi}=0$, we can derive the evolution equation for the angular momentum $J_{\text {Bondi }}^{\phi}(u)$ by gravitational waves as

$$
\frac{d}{d u} J_{\text {Bondi }}^{\phi}(u)=-\frac{1}{4 \pi} \int_{S^{3}}\left[\left(\frac{\partial j^{\phi}}{\partial u}\right)_{\text {radiation }}+\left(\frac{\partial j^{\phi}}{\partial u}\right)_{\text {total derivative }}\right] d \Omega,
$$

where $\left(\partial j^{\phi} / \partial u\right)_{\text {radiation }}$ is the radiation part given by

$$
\begin{aligned}
\left(\frac{\partial j^{\phi}}{\partial u}\right)_{\text {radiation }}= & -\frac{1}{4} \frac{\partial C_{11}}{\partial \phi} \frac{\partial C_{11}}{\partial u}-\frac{1}{8} \frac{\partial C_{21}}{\partial \phi} \frac{\partial C_{11}}{\partial u}-\frac{1}{8} \frac{\partial C_{11}}{\partial \phi} \frac{\partial C_{21}}{\partial u}-\frac{1}{4} \frac{\partial C_{21}}{\partial \phi} \frac{\partial C_{21}}{\partial u}-\frac{1}{4} \frac{\partial D_{21}}{\partial \phi} \frac{\partial D_{21}}{\partial u} \\
& +\frac{1}{10} \tan \theta \frac{\partial D_{31}}{\partial u}\left(\frac{\partial C_{11}}{\partial \psi}+\frac{\partial C_{21}}{\partial \psi}\right)+\frac{3}{20} \tan \theta \frac{\partial D_{31}}{\partial \psi} \frac{\partial C_{11}}{\partial u}+\frac{2}{5} \tan \theta \frac{\partial D_{31}}{\partial \psi} \frac{\partial C_{21}}{\partial u} \\
& -\frac{1}{4} \frac{\partial D_{11}}{\partial \phi} \frac{\partial D_{11}}{\partial u}+\frac{3}{20} \tan \theta \frac{\partial D_{11}}{\partial u} \frac{\partial D_{21}}{\partial \psi}-\frac{3}{20} \tan \theta \frac{\partial D_{11}}{\partial \psi} \frac{\partial D_{21}}{\partial u}-\frac{1}{4} \tan \theta \frac{\partial C_{11}}{\partial \psi} \frac{\partial D_{31}}{\partial u} \\
& -\frac{1}{10} \tan \theta \frac{\partial C_{21}}{\partial u} \frac{\partial D_{31}}{\partial \psi}-\frac{2}{5} \tan \theta \frac{\partial D_{31}}{\partial u} \frac{\partial C_{21}}{\partial \psi}-\frac{1}{4} \frac{\partial D_{31}}{\partial u} \frac{\partial D_{31}}{\partial \phi} \\
& +\frac{3}{20 \cos ^{2} \theta} \frac{\partial D_{31}}{\partial u} \frac{\partial}{\partial \theta}\left(\sin \theta \cos ^{2} \theta D_{21}\right)-\frac{3}{20} \cos \theta \frac{\partial}{\partial \theta}\left(\tan \theta D_{31}\right) \frac{\partial D_{21}}{\partial u} \\
& +\frac{3}{20 \cos ^{2} \theta} \frac{\partial D_{11}}{\partial u} \frac{\partial}{\partial \theta}\left(\sin \theta \cos ^{2} \theta C_{11}\right)-\frac{3}{20} \cos \theta \frac{\partial C_{11}}{\partial u} \frac{\partial}{\partial \theta}\left(\tan \theta D_{11}\right) \\
& +\frac{3}{20 \sin \theta \cos \theta} \frac{\partial C_{21}}{\partial u} \frac{\partial}{\partial \theta}\left(\sin ^{2} \theta \cos \theta D_{11}\right) \\
& -\frac{3}{20} \sin \theta \frac{\partial C_{21}}{\partial \theta} \frac{\partial D_{11}}{\partial u}-\frac{3}{20 \cos \theta} \frac{\partial}{\partial u}\left(C_{21} D_{11}\right),
\end{aligned}
$$

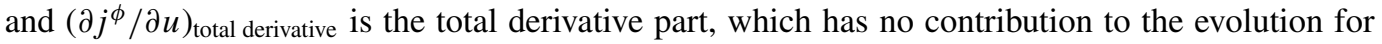
the angular momentum by gravitational waves. The explicit form is given by

$$
\begin{aligned}
\left(\frac{\partial j^{\phi}}{\partial u}\right)_{\text {total derivative }}= & \frac{1}{4}\left[3 \sin ^{2} \theta U_{2}^{\phi}-\tan ^{2} \theta \frac{\partial^{2}}{\partial \psi^{2}} U_{2}^{\phi}-\cot \theta \frac{\partial}{\partial \phi} U_{2}^{\theta}\right. \\
& -\tan \theta \frac{\partial}{\partial \phi} U_{2}^{\theta}+\frac{\partial^{2}}{\partial \phi \partial \theta} U_{2}^{\theta}+\frac{\partial}{\partial \phi} m \\
& +\frac{\partial^{2}}{\partial \phi \partial \psi} U_{2}^{\psi}-\frac{1}{\sin \theta \cos \theta} \frac{\partial}{\partial \theta}\left(\sin ^{3} \theta \cos \theta \frac{\partial}{\partial \theta} U_{2}^{\phi}\right)
\end{aligned}
$$




$$
\begin{aligned}
& +\frac{1}{\sin \theta \cos \theta} \frac{\partial}{\partial \theta}\left(\sin ^{2} \theta \cos \theta \frac{\partial D_{14}}{\partial u}\right) \\
& +\tan \theta \frac{\partial}{\partial \psi} \frac{\partial D_{34}}{\partial u}+\frac{\partial}{\partial \phi} \frac{\partial C_{24}}{\partial u}-\frac{\partial}{\partial \phi} \frac{\partial B_{1}}{\partial u} \\
& -\frac{3}{5} \tan \theta \frac{\partial}{\partial \psi}\left(D_{31} \frac{\partial C_{11}}{\partial u}\right)-\frac{8}{5} \tan \theta \frac{\partial}{\partial \psi}\left(D_{31} \frac{\partial C_{21}}{\partial u}\right) \\
& +\frac{3}{5} \frac{\partial}{\partial \phi}\left(C_{21} \frac{\partial C_{21}}{\partial u}\right)-\tan \theta \frac{\partial}{\partial \psi}\left(D_{21} \frac{\partial D_{11}}{\partial u}\right) \\
& -\frac{2}{5} \frac{\partial}{\partial \phi}\left(D_{11} \frac{\partial D_{11}}{\partial u}\right)+\frac{3}{5} \tan \theta \frac{\partial}{\partial \psi}\left(D_{11} \frac{\partial D_{21}}{\partial u}\right) \\
& +\tan \theta \frac{\partial}{\partial \psi}\left(C_{11} \frac{\partial D_{31}}{\partial u}\right)+\frac{8}{5} \tan \theta \frac{\partial}{\partial \psi}\left(C_{21} \frac{\partial D_{31}}{\partial u}\right)-\frac{2}{5} \frac{\partial}{\partial \phi}\left(D_{31} \frac{\partial D_{31}}{\partial u}\right) \\
& -\frac{1}{\sin \theta \cos \theta} \frac{\partial}{\partial \theta}\left(\sin ^{2} \theta \cos \theta D_{21} \frac{\partial D_{31}}{\partial u}\right) \\
& +\frac{3}{\sin \theta \cos \theta} \frac{\partial}{\partial \theta}\left(\sin ^{2} \theta \cos \theta C_{11} \frac{\partial D_{11}}{\partial u}\right) \\
& +\frac{3 \sin \theta \cos \theta}{5 \sin \theta \cos \theta} \frac{\partial}{\partial \theta}\left(\sin ^{2} \theta \cos \theta D_{31} \frac{\partial D_{21}}{\partial u}\right) \\
& -\frac{1}{\sin \theta \cos \theta} \frac{\partial}{\partial \theta}\left(\sin ^{2} \theta \cos \theta D_{11} \frac{\partial C_{21}}{\partial u}\right)
\end{aligned}
$$

Then the evolution equation for angular momentum $J_{\text {Bondi }}^{\phi}(u)$ is expressed as

$$
\frac{d}{d u} J_{\text {Bondi }}^{\phi}(u)=-\frac{1}{4 \pi} \int_{S^{3}}\left(\frac{\partial j^{\phi}}{\partial u}\right)_{\text {radiation }} d \Omega .
$$

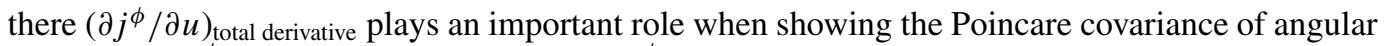
momentum $J_{\text {Bondi }}^{\phi}$ in Sec. IV. Note that $d J_{\text {Bondi }}^{\phi} / d u=0$ if there is no gravitational wave, i.e., $\partial h_{A B}^{(1)} / \partial u=0$.

Similarly, from $R_{u \psi}=0$, we can see

$$
\frac{d}{d u} J_{\text {Bondi }}^{\psi}(u)=-\frac{1}{4 \pi} \int_{S^{3}}\left[\left(\frac{\partial j^{\psi}}{\partial u}\right)_{\text {radiation }}+\left(\frac{\partial j^{\psi}}{\partial u}\right)_{\text {total derivative }}\right] d \Omega,
$$

where

$$
\begin{aligned}
\left(\frac{\partial j^{\psi}}{\partial u}\right)_{\text {radiation }}= & -\frac{1}{4} \frac{\partial C_{11}}{\partial \psi} \frac{\partial C_{11}}{\partial u}-\frac{1}{4} \frac{\partial C_{21}}{\partial \psi} \frac{\partial C_{21}}{\partial u} \\
& -\frac{1}{8} \frac{\partial C_{21}}{\partial \psi} \frac{\partial C_{11}}{\partial u}-\frac{1}{8} \frac{\partial C_{11}}{\partial \psi} \frac{\partial C_{21}}{\partial u}-\frac{3}{20} \cos \theta \frac{\partial D_{21}}{\partial \theta} \frac{\partial C_{11}}{\partial u} \\
& -\frac{1}{4} \cos \theta \frac{\partial C_{11}}{\partial \theta} \frac{\partial D_{21}}{\partial u}-\frac{1}{4} \cot \theta \frac{\partial C_{21}}{\partial \phi} \frac{\partial D_{31}}{\partial u} \\
& +\frac{3}{20} \cot \theta \frac{\partial C_{21}}{\partial \phi} \frac{\partial D_{31}}{\partial u}-\frac{3}{20} \cot \theta \frac{\partial D_{31}}{\partial \phi} \frac{\partial C_{21}}{\partial u}
\end{aligned}
$$




$$
\begin{aligned}
& -\frac{1}{4} \frac{\partial D_{11}}{\partial \psi} \frac{\partial D_{11}}{\partial u}+\frac{3}{20} \cot \theta \frac{\partial D_{11}}{\partial \phi} \frac{\partial D_{21}}{\partial u} \\
& -\frac{3}{20} \cot \theta \frac{\partial D_{21}}{\partial \phi} \frac{\partial D_{11}}{\partial u}-\frac{1}{4} \frac{\partial D_{21}}{\partial \psi} \frac{\partial D_{21}}{\partial u}-\frac{1}{4} \frac{\partial D_{31}}{\partial \psi} \frac{\partial D_{31}}{\partial u} \\
& -\frac{3}{20} \cot \theta \frac{\partial D_{31}}{\partial \phi} \frac{\partial}{\partial u}\left(C_{11}+C_{21}\right)+\frac{3}{20} \cot \theta \frac{\partial C_{11}}{\partial \phi} \frac{\partial D_{31}}{\partial u}+\frac{2}{5} \cot \theta \frac{\partial C_{21}}{\partial \phi} \frac{\partial D_{31}}{\partial u} \\
& +\frac{3}{20 \sin \theta \cos \theta} \frac{\partial}{\partial \theta}\left(\sin \theta \cos ^{2} \theta C_{11}\right) \frac{\partial D_{21}}{\partial u}+\frac{3}{20 \sin ^{2} \theta} \frac{\partial}{\partial \theta}\left(\sin ^{2} \theta \cos \theta D_{11}\right) \frac{\partial D_{31}}{\partial u} \\
& -\frac{3}{20} \sin \theta \frac{\partial}{\partial \theta}\left(\cot \theta D_{31}\right) \frac{\partial D_{11}}{\partial u}+\frac{2}{5} \cos \theta \frac{\partial C_{11}}{\partial \theta} \frac{\partial D_{21}}{\partial u}+\frac{3}{20} \cos \theta \frac{\partial C_{21}}{\partial \theta} \frac{\partial D_{21}}{\partial u} \\
& -\frac{3}{20 \sin \theta} \frac{\partial}{\partial u}\left(D_{21} C_{21}\right)-\frac{3}{20 \sin \theta \cos \theta} \frac{\partial}{\partial \theta}\left(\sin \theta \cos ^{2} \theta D_{21}\right) \frac{\partial}{\partial u}\left(C_{11}+C_{21}\right),
\end{aligned}
$$

and

$$
\begin{aligned}
&\left(\frac{\partial j^{\psi}}{\partial u}\right)_{\text {total derivative }}= \frac{3}{4} \cos ^{2} \theta U_{2}^{\psi}+\frac{\csc 2 \theta}{2} \frac{\partial}{\partial \psi} U_{2}^{\theta}+\frac{1}{4} \frac{\partial^{2}}{\partial \phi \partial \psi} U_{2}^{\phi} \\
&-\frac{\cot ^{2} \theta}{4} \frac{\partial^{2}}{\partial \phi^{2}} U_{2}^{\psi}+\frac{1}{4} \frac{\partial^{2}}{\partial \theta \partial \psi} U_{2}^{\theta}+\frac{1}{4} \frac{\partial m}{\partial \psi} \\
&-\frac{1}{4 \sin \theta \cos \theta} \frac{\partial}{\partial \theta}\left(\sin \theta \cos ^{3} \theta \frac{\partial}{\partial \theta} U_{2}^{\psi}\right) \\
&+\frac{1}{4 \sin \theta \cos \theta}\left(\frac{\partial}{\partial \theta} \sin \theta \cos ^{2} \theta \frac{\partial D_{24}}{\partial u}\right)+\frac{\cot \theta}{4} \frac{\partial^{2}}{\partial \phi \partial u} D_{34} \\
&-\frac{1}{4} \frac{\partial^{2}}{\partial \psi \partial u}\left(C_{14}+C_{24}\right)-\frac{1}{4} \frac{\partial^{2}}{\partial \psi \partial u} B_{1}+\frac{3}{20} \frac{\partial}{\partial \psi}\left(\left(C_{11}+C_{21}\right) \frac{\partial}{\partial u}\left(C_{11}+C_{21}\right)\right) \\
&+\frac{1}{4} \frac{\partial}{\partial \phi}\left(\cot \theta D_{31} \frac{\partial}{\partial u} C_{11}\right)+\frac{2}{5} \frac{\partial}{\partial \phi}\left(\cot \theta D_{31} \frac{\partial}{\partial u} C_{21}\right)+\frac{1}{2} \frac{\partial}{\partial \psi}\left(D_{11} \frac{\partial}{\partial u} D_{11}\right) \\
&+\frac{3}{20} \frac{\partial}{\partial \phi}\left(\cot \theta D_{21} \frac{\partial}{\partial u} D_{11}\right)+\frac{2}{5} \frac{\partial}{\partial \psi}\left(D_{21} \frac{\partial}{\partial u} D_{21}\right)-\frac{1}{4} \frac{\partial}{\partial \phi}\left(\cot \theta D_{11} \frac{\partial}{\partial u} D_{21}\right) \\
&+\frac{2}{5} \frac{\partial}{\partial \psi}\left(D_{31} \frac{\partial}{\partial u} D_{31}\right)-\frac{3}{20} \frac{\partial}{\partial \phi}\left(\cot \theta C_{11} \frac{\partial}{\partial u} D_{31}\right)-\frac{2}{5} \frac{\partial}{\partial \phi}\left(\cot \theta C_{21} \frac{\partial}{\partial u} D_{31}\right) \\
&+\frac{2}{5 \sin \theta \cos \theta} \frac{\partial}{\partial \theta}\left(\sin \theta \cos ^{2} \theta D_{21} \frac{\partial}{\partial u} C_{11}\right) \\
&+\frac{1}{4 \sin \theta \cos \theta} \frac{\partial}{\partial \theta}\left(\sin \theta \cos ^{2} \theta D_{21} \frac{\partial}{\partial u} C_{21}\right) \\
&+\frac{3}{20 \sin \theta \cos \theta} \frac{\partial}{\partial \theta}\left(\sin \theta \cos ^{2} \theta D_{31} \frac{\partial}{\partial u} D_{11}\right) \\
&-\frac{2}{5 \sin \theta \cos \theta} \frac{\partial}{\partial \theta}\left(\sin \theta \cos ^{2} \theta C_{11} \frac{\partial}{\partial u} D_{21}\right) \\
& \frac{3}{4 \sin \theta \cos \theta} \frac{\partial}{\partial \theta}\left(\sin \theta \cos ^{2} \theta D_{11} \frac{\partial}{\partial u} D_{31}\right) \cdot \\
&\left.\partial \sin \theta \cos ^{2} \theta C_{21} \frac{\partial}{\partial u} D_{21}\right) \\
& \\
&+43) \\
& \\
&
\end{aligned}
$$


Then the evolution equation for the angular momentum $J_{\text {Bondi }}^{\psi}(u)$ is given by

$$
\frac{d}{d u} J_{\text {Bondi }}^{\psi}(u)=-\frac{1}{4 \pi} \int_{S^{3}}\left(\frac{\partial j^{\psi}}{\partial u}\right)_{\text {radiation }} d \Omega .
$$

\section{ASYMPTOTIC SYMMETRY}

In this section, we consider asymptotic symmetry at null infinity. Following our previous work, ${ }^{16}$ we first discuss the asymptotic symmetry. See Ref. 16 for the details. Then we will show the Poincare covariance of the Bondi mass and angular momentum.

\section{A. Preliminary}

Asymptotic symmetry is defined as the transformation group which preserve the boundary conditions of Eqs. (5)-(9) at null infinity. By infinitesimal transformations $\xi^{a}$, the metric is transformed as $g_{a b} \rightarrow g_{a b}+\delta g_{a b}$, where

$$
\delta g_{a b}=\nabla_{a} \xi_{b}+\nabla_{b} \xi_{a}
$$

To preserve the boundary conditions, the metric variation $\delta g_{a b}$ should satisfy the following conditions:

$$
\begin{gathered}
\delta g_{r r}=0, \delta g_{r A}=0, g^{A B} \delta g_{A B}=0, \\
\delta g_{u u}=O\left(r^{-3 / 2}\right), \delta g_{u A}=O\left(r^{-1 / 2}\right), \delta g_{A B}=O\left(r^{1 / 2}\right) .
\end{gathered}
$$

The conditions of Eq. (46) comes from the definition of the Bondi coordinates. Next the conditions of Eq. (47) are required from the behavior of gravitational fields near null infinity. From the condition of Eq. (46), we can see that the infinitesimal transformation $\xi^{a}$ can be written as

$$
\begin{gathered}
\xi_{r}=f\left(u, x^{A}\right) e^{B}, \\
\xi_{B} g^{A B}=f^{A}\left(u, x^{A}\right)-f\left(u, x^{A}\right) U^{A}+\int_{r}^{\infty} d r^{\prime} e^{B} \frac{\partial f}{\partial x^{B}} g^{A B}, \\
\xi_{u}=-\frac{r e^{B}}{3}\left(-\frac{\partial \xi_{A}}{\partial x^{B}}+\xi_{C} \Gamma_{A B}^{C}+\xi_{r} \Gamma_{A B}^{r}\right) g^{A B},
\end{gathered}
$$

where $f\left(u, x^{A}\right)$ and $f^{A}\left(u, x^{A}\right)$ are functions which satisfy

$$
\begin{gathered}
\frac{\partial f^{A}}{\partial u}=0, \\
\mathcal{D}_{A} f_{B}+\mathcal{D}_{B} f_{A}=-2 \frac{\partial f}{\partial u} h_{A B}^{(0)}, \\
\mathcal{D}_{A} \mathcal{D}_{B} f=\frac{1}{3} \mathcal{D}^{2} f h_{A B}^{(0)} .
\end{gathered}
$$

In the above, $\mathcal{D}_{A}$ is the covariant derivative with respect to $h_{A B}^{(0)}$. From Eqs. (51) and (52), we can see that $f^{A}$ should be conformal Killing vector on $S^{3}$. Since such transformation group (conformal Killing group on $S^{3}$ ) is isomorphic to the Lorentz group, $f^{A}$ corresponds to the generator of the Lorentz transformations. The function $f$ can be written by $f=-F\left(x^{A}\right) u / 3+\alpha\left(x^{A}\right)$, where 
$F\left(x^{A}\right)=\mathcal{D}_{A} f^{A}$. We would guess that $\alpha\left(x^{A}\right)$ is the generator of translations. From Eq. (53), we can see that the function $\alpha\left(x^{A}\right)$ can be written as

$$
\alpha\left(x^{A}\right)=a_{u}+a_{x} \sin \theta \cos \phi+a_{y} \sin \theta \sin \phi+a_{z} \cos \theta \cos \psi+a_{w} \cos \theta \sin \psi .
$$

This $\alpha$ has only five parameters which correspond to five directions of translations. Thus, the asymptotic symmetry generated by $\xi$ at null infinity is the Poincare group which is semidirect of the Lorentz group and the translation group. On the other hand, in four dimensions, there is no conditions on $f$ like Eq. (53). This means that $\alpha\left(x^{A}\right)$ in $f$ generates the infinite dimensional translation group, that is, the supertranslation group in four dimensions.

In our previous work, ${ }^{16}$ we have not discussed the asymptotic symmetry in terms of global charges. Hence we will address this point in Secs. IV B and IV C.

\section{B. Poincare covariance of the Bondi mass}

Asymptotic quantities such as the Bondi mass and angular momentum should be global charges associated with the asymptotic symmetry. To confirm this, in this section, we will consider translations generated by $\xi$ with

$$
f=\alpha\left(x^{A}\right), f^{A}=0 .
$$

This transformation implies $u \rightarrow u-f\left(x^{A}\right)$.

The global charges associated with asymptotic symmetry (the Poincare group) are energymomentum vector $P_{a}$ and angular momentum $M_{a b}$. And they should be transformed under translations of Eq. (55) as

$$
P_{a} \rightarrow P_{a}, \quad M_{a b} \rightarrow M_{a b}+2 P_{[a} \omega_{b]},
$$

where $f=\hat{x}^{a} \omega_{a}$ and $\hat{x}^{a}=\left(1, \hat{x}^{i}\right)$. However, we are considering dynamical space-times which has no exact timelike Killing vector. This means that the quantities $P_{a}$ and $M_{a b}$ would change due to gravitational waves under translations $\left(u \rightarrow u-f\left(x^{A}\right)\right)$. Then, the expected transformations of $P_{a}$ and $M_{a b}$ under translation of Eq. (55) are

$$
\begin{aligned}
P_{a}(u) & \rightarrow P_{a}(u-f)=P_{a}(u)-\left(f \frac{d}{d u} P_{a}(u)\right)_{\text {radiation }}, \\
M_{a b}(u) & \rightarrow M_{a b}(u-f)+2 P_{[a}(u) \omega_{b]}=M_{a b}(u)+2 P_{[a}(u) \omega_{b]}-\left(f \frac{d}{d u} M_{a b}(u)\right)_{\text {radiation }} .
\end{aligned}
$$

In Eq. (58), note that the argument of the second term $2 P_{[a} \omega_{b]}$ is $u$, not $u-f$. This is because this term corresponds to the orbital angular momentum generated by translations. The purpose of this section is to confirm this property. First, we check the relation of Eq. (57). Under the translations of Eq. (55), $m\left(u, x^{A}\right)$ in $g_{u u}$ transforms as

$$
m \rightarrow m-\alpha\left(x^{A}\right) \frac{\partial m}{\partial u}-\frac{2}{3} \frac{\partial \alpha}{\partial \theta} \frac{\partial U_{2}^{\theta}}{\partial u}-\frac{2}{3} \frac{\partial \alpha}{\partial \phi} \frac{\partial U_{2}^{\phi}}{\partial u}-\frac{2}{3} \frac{\partial \alpha}{\partial \psi} \frac{\partial U_{2}^{\psi}}{\partial u}+\text { (total derivative terms). }
$$

Here we do not write down the explicit form of the total derivative terms in this equation because it is slightly complicated and not important for our purpose. From the above equation, we find that the Bondi energy momentum $P_{\text {Bondi }}^{a}$ transforms under the translations as

$$
\begin{aligned}
& P_{\text {Bondi }}^{a} \rightarrow P_{\text {Bondi }}^{a}+\frac{3}{16 \pi} \int_{S^{3}} \hat{x}^{a} {\left[-\alpha\left(x^{A}\right) \frac{\partial m}{\partial u}-\frac{2}{3} \frac{\partial \alpha}{\partial \theta} \frac{\partial U_{2}^{\theta}}{\partial u}-\frac{2}{3} \frac{\partial \alpha}{\partial \phi} \frac{\partial U_{2}^{\phi}}{\partial u}-\frac{2}{3} \frac{\partial \alpha}{\partial \psi} \frac{\partial U_{2}^{\psi}}{\partial u}\right] d \Omega } \\
&=P_{\text {Bondi }}^{a}+\frac{1}{16 \pi} \int_{S^{3}} \hat{x}^{a}\{\alpha {\left[\left(\frac{\partial C_{11}}{\partial u}\right)^{2}+\frac{\partial C_{11}}{\partial u} \frac{\partial C_{21}}{\partial u}+\left(\frac{\partial C_{21}}{\partial u}\right)^{2}\right.} \\
&\left.+\left(\frac{\partial D_{11}}{\partial u}\right)^{2}+\left(\frac{\partial D_{21}}{\partial u}\right)^{2}+\left(\frac{\partial D_{31}}{\partial u}\right)^{2}\right]
\end{aligned}
$$




$$
\begin{gathered}
\left.-\left[\frac{2}{\sin \theta \cos \theta} \frac{\partial}{\partial \theta}\left(\alpha \sin \theta \cos \theta \frac{\partial U_{2}^{\theta}}{\partial u}\right)+2 \frac{\partial^{2}}{\partial \phi \partial u}\left(\alpha U_{2}^{\phi}\right)+2 \frac{\partial^{2}}{\partial \psi \partial u}\left(\alpha U_{2}^{\psi}\right)\right]\right\} d \Omega \\
=P_{\text {Bondi }}^{a}+\frac{1}{16 \pi} \int_{S^{3}} \alpha \hat{x}^{a}\left\{\left(\frac{\partial C_{11}}{\partial u}\right)^{2}+\frac{\partial C_{11}}{\partial u} \frac{\partial C_{21}}{\partial u}+\left(\frac{\partial C_{21}}{\partial u}\right)^{2}\right. \\
\left.+\left(\frac{\partial D_{11}}{\partial u}\right)^{2}+\left(\frac{\partial D_{21}}{\partial u}\right)^{2}+\left(\frac{\partial D_{31}}{\partial u}\right)^{2}\right\} d \Omega
\end{gathered}
$$

In the above, we used the fact that $U_{2}^{A}$ are time independent. This can be shown using Eqs. (20)-(22) and $\partial h_{A B}^{(2)} / \partial u=0$. As seen in Sec. III, the integral part in Eq. (60) can be interpreted as the energy momentum loss by gravitational wave $\left(f d P_{a} / d u\right)_{\text {radiation. Then, the Bondi }}$ energy momentum satisfies the Poincare covariance of Eq. (57).

\section{Poincare covariance of the Bondi angular momentum}

Next, we show the Poincare covariance of the Bondi angular momentum $J_{\text {Bondi }}^{\phi}$. By the translations of $\mathrm{Eq}(55), j^{\phi}$ is transformed as

$$
j^{\phi} \rightarrow j^{\phi}-\alpha\left(x^{A}\right) \frac{\partial j^{\phi}}{\partial u}+\left(\delta j^{\phi}\right)_{\text {nonradiation }}+(\text { total derivative terms }),
$$

where

$$
\begin{aligned}
\left(\delta j^{\phi}\right)_{\text {nonradiation }}= & \frac{2}{3} \frac{\partial^{2} \alpha}{\partial \theta \partial \phi} U_{2}^{\theta}-\frac{1}{3} \frac{\partial \alpha}{\partial \theta} \frac{\partial U_{2}^{\theta}}{\partial \phi}+\frac{1}{3} \tan ^{2} \theta \frac{\partial^{2} \alpha}{\partial \psi^{2}} U_{2}^{\phi}+\frac{\partial^{2} \alpha}{\partial \phi^{2}} U_{2}^{\phi} \\
& +\frac{1}{6}(6+8 \cos 2 \theta) \tan \theta \frac{\partial \alpha}{\partial \theta} U_{2}^{\phi} \\
& +\frac{1}{3} \sin ^{2} \theta \frac{\partial^{2} \alpha}{\partial \theta^{2}} U_{2}^{\phi}+\tan ^{2} \theta \frac{\partial \alpha}{\partial \psi} \frac{\partial U_{2}^{\phi}}{\partial \psi}+\frac{2}{3} \frac{\partial \alpha}{\partial \phi} \frac{\partial U_{2}^{\phi}}{\partial \phi} \\
& +\sin ^{2} \theta \frac{\partial \alpha}{\partial \theta} \frac{\partial U_{2}^{\phi}}{\partial \theta}+\frac{2}{3} \frac{\partial^{2} \alpha}{\partial \phi \partial \psi} U_{2}^{\psi}-\frac{1}{3} \frac{\partial \alpha}{\partial \psi} \frac{\partial U_{2}^{\psi}}{\partial \phi} \\
& -m \frac{\partial \alpha}{\partial \phi}+\frac{1}{4} \frac{\partial \alpha}{\partial \phi} \frac{\partial B_{1}}{\partial u}-\frac{1}{4} \frac{\partial \alpha}{\partial \phi} \frac{\partial C_{24}}{\partial u}-\frac{1}{4} \frac{\partial \alpha}{\partial \theta} \frac{\partial D_{14}}{\partial u} \\
& -\frac{1}{4} \tan \theta \frac{\partial \alpha}{\partial \psi} \frac{\partial D_{34}}{\partial u}+\frac{3}{20} \tan \theta D_{31} \frac{\partial \alpha}{\partial \psi} \frac{\partial C_{11}}{\partial u} \\
& -\frac{3}{20} \sin \theta D_{11} \frac{\partial \alpha}{\partial \theta} \frac{\partial C_{11}}{\partial u}+\frac{2}{5} \tan \theta D_{31} \frac{\partial \alpha}{\partial \psi} \frac{\partial C_{21}}{\partial u} \\
& -\frac{3}{20} C_{21} \frac{\partial \alpha}{\partial \phi} \frac{\partial C_{21}}{\partial u}+\frac{1}{4} \sin \theta D_{11} \frac{\partial \alpha}{\partial \theta} \frac{\partial C_{21}}{\partial u} \\
& +\frac{1}{4} \tan \theta D_{21} \frac{\partial \alpha}{\partial \psi} \frac{\partial D_{11}}{\partial u}+\frac{1}{10} D_{11} \frac{\partial \alpha}{\partial \phi} \frac{\partial D_{11}}{\partial u} \\
& +\frac{1}{4} \sin \theta C_{11} \frac{\partial \alpha}{\partial \theta} \frac{\partial D_{11}}{\partial u}-\frac{3}{20} \sin \theta C_{21} \frac{\partial \alpha}{\partial \theta} \frac{\partial D_{11}}{\partial u} \\
& -\frac{3}{20} \tan \theta D_{11} \frac{\partial \alpha}{\partial \psi} \frac{\partial D_{21}}{\partial u}-\frac{3}{20} \sin \theta D_{31} \frac{\partial \alpha}{\partial \theta} \frac{\partial D_{21}}{\partial u} \\
& -\frac{1}{4} \tan \theta C_{11} \frac{\partial \alpha}{\partial \psi} \frac{\partial D_{31}}{\partial u}-\frac{2}{5} \tan \theta C_{21} \frac{\partial \alpha}{\partial \psi} \frac{\partial D_{31}}{\partial u} \\
& +\frac{1}{10} D_{31} \frac{\partial \alpha}{\partial \phi} \frac{\partial D_{31}}{\partial u}+\frac{1}{4} \sin \theta D_{21} \frac{\partial \alpha}{\partial \theta} \frac{\partial D_{31}}{\partial u} . \\
\frac{1}{\partial u} &
\end{aligned}
$$


Then, under the translations of Eq. (55), the Bondi angular momentum at null infinity transforms as

$$
\begin{aligned}
J_{\text {Bondi }}^{\phi} \rightarrow & J_{\text {Bondi }}^{\phi}-\frac{1}{4 \pi} \int_{S^{3}}\left[-\alpha\left(x^{A}\right) \frac{\partial j^{\phi}}{\partial u}+\left(\delta j^{\phi}\right)_{\text {nonradiation }}\right] d \Omega \\
& =J_{\text {Bondi }}^{\phi}-\frac{1}{4 \pi} \int_{S^{3}}\left[-\alpha\left(x^{A}\right)\left(\frac{\partial j^{\phi}}{\partial u}\right)_{\text {radiation }}-\alpha\left(x^{A}\right)\left(\frac{\partial j^{\phi}}{\partial u}\right)_{\text {total derivative }}+\left(\delta j^{\phi}\right)_{\text {nonradiation }}\right] d \Omega .
\end{aligned}
$$

Now, the straightforward calculations tells us

$-\alpha\left(x^{A}\right)\left(\frac{\partial j^{\phi}}{\partial u}\right)_{\text {total derivative }}+\left(\delta j^{\phi}\right)_{\text {nonradiation }}=-\frac{3}{4} m \frac{\partial \alpha}{\partial \phi}+(\text { supermomentum })_{\phi}+($ total derivative $)$,

where supermomentum term is given by

$$
\begin{aligned}
\text { (supermomentum })_{\phi}= & \frac{\alpha}{4}\left[5 \cot \theta \frac{\partial}{\partial \phi} U_{2}^{\theta}-3 \tan \theta \frac{\partial}{\partial \phi} U_{2}^{\theta}+3 \frac{\partial^{2}}{\partial \theta \partial \phi} U_{2}^{\theta}\right] \\
& +\frac{\alpha}{12}\left[23 \sin ^{2} \theta U_{2}^{\phi}-32 \cos ^{2} \theta U_{2}^{\phi}-31 \cos \theta \sin \theta \frac{\partial}{\partial \theta} U_{2}^{\phi}+5 \frac{\sin ^{3} \theta}{\cos \theta} \frac{\partial}{\partial \theta} U_{2}^{\phi}\right. \\
& \left.-5 \sin ^{2} \theta \frac{\partial^{2}}{\partial \theta^{2}} U_{2}^{\phi}+4 \frac{\partial^{2}}{\partial \phi^{2}} U_{2}^{\phi}-5 \tan ^{2} \theta \frac{\partial^{2}}{\partial \psi^{2}} U_{2}^{\phi}\right] \\
& +\frac{3}{4} \alpha \frac{\partial^{2}}{\partial \phi \partial \psi} U_{2}^{\psi} .
\end{aligned}
$$

In the above $\alpha\left(x^{A}\right)$ has only $l=0$ and $l=1$ modes of scalar harmonics on $S^{3}$. Using the solution of the Einstein equations (20)-(22), then, we can show that these supermomentum terms become total derivative. Then, taking these results altogether, we see

$$
J_{\text {Bondi }}^{\phi} \rightarrow J_{\text {Bondi }}^{\phi}-\frac{1}{4 \pi} \int_{S^{3}}\left[-\alpha\left(x^{A}\right)\left(\frac{\partial j^{\phi}}{\partial u}\right)_{\text {radiation }}\right] d \Omega+\frac{3}{16 \pi} \int_{S^{3}} m\left(u, x^{A}\right) \frac{\partial \alpha}{\partial \phi} d \Omega .
$$

This transformation is equivalent to $M_{\hat{x} \hat{y}} \rightarrow M_{\hat{x} \hat{y}}+2 P_{[\hat{x}} \omega_{\hat{y}]}-\left(f d M_{\hat{x} \hat{y}} / d u\right)_{\text {radiation. }}$ This stands for the Poincare covariance of the Bondi angular momentum. In the same way, we can show the Poincare covariance of angular momentum $J_{\text {Bondi }}^{\psi}$. Under translations, $J_{\text {Bondi }}^{\psi}$ transforms as

$$
\begin{aligned}
J_{\text {Bondi }}^{\psi} & \rightarrow J_{\text {Bondi }}^{\psi}-\frac{1}{4 \pi} \int_{S^{3}}\left[-\alpha\left(x^{A}\right)\left(\frac{\partial j^{\psi}}{\partial u}\right)_{\text {radiation }}+(\text { supermomentum })_{\psi}\right] d \Omega \\
& +\frac{3}{16 \pi} \int_{S^{3}} m\left(u, x^{A}\right) \frac{\partial \alpha}{\partial \psi} d \Omega,
\end{aligned}
$$

where the supermomentum term is

$$
\begin{aligned}
\text { (supermomentum })_{\psi}= & \frac{\alpha}{4}\left[3 \cot \theta \frac{\partial}{\partial \psi} U_{2}^{\theta}-5 \tan \theta \frac{\partial}{\partial \psi} U_{2}^{\theta}+3 \frac{\partial^{2}}{\partial \theta \psi} U_{2}^{\theta}\right]-\frac{3}{4} \alpha \frac{\partial^{2}}{\partial \phi \partial \psi} U_{2}^{\phi} \\
& +\frac{\alpha}{12}\left[-32 \sin ^{2} \theta U_{2}^{\psi}+23 \cos ^{2} \theta U_{2}^{\psi}+31 \cos \theta \sin \theta \frac{\partial}{\partial \theta} U_{2}^{\psi}-5 \frac{\cos ^{3} \theta}{\sin \theta} \frac{\partial}{\partial \theta} U_{2}^{\psi}\right. \\
& \left.-5 \cos ^{2} \theta \frac{\partial^{2}}{\partial \theta^{2}} U_{2}^{\psi}+4 \frac{\partial^{2}}{\partial \psi^{2}} U_{2}^{\psi}-5 \cot ^{2} \theta \frac{\partial^{2}}{\partial \phi^{2}} U_{2}^{\phi}\right] .
\end{aligned}
$$

For the $l=0$ and $l=1$ modes in $\alpha$, these supermomentum terms become total derivative. Finally, we obtain the Poincare transformations of angular momentum as

$$
J_{\text {Bondi }}^{\psi} \rightarrow J_{\text {Bondi }}^{\psi}-\frac{1}{4 \pi} \int_{S^{3}}\left[-\alpha\left(x^{A}\right)\left(\frac{\partial j^{\psi}}{\partial u}\right)_{\text {radiation }}\right] d \Omega+\frac{3}{16 \pi} \int_{S^{3}} m\left(u, x^{A}\right) \frac{\partial \alpha}{\partial \psi} d \Omega .
$$


This transformation is equivalent to $M_{\hat{z} \hat{w}} \rightarrow M_{\hat{z} \hat{w}}+2 P_{[\hat{z}} \omega_{\hat{w}]}-\left(f d M_{\hat{z} \hat{w}} / d u\right)_{\text {radiation }}$.

Since $\alpha$ contains all $l$ mode of scalar harmonics on $S^{2}$ in four dimensions, the supermomentum does not vanish in general. Thus we cannot show the Poincare covariance of angular momentum in four dimensions (see the Appendix for the details).

\section{SUMMARY AND DISCUSSION}

In this paper, we defined the Bondi angular momentum at null infinity in five-dimensions and showed the Poincare covariance of the Bondi mass and angular momentum. In addition, we successfully confirmed the Bondi mass loss and angular momentum loss/gain due to gravitational wave.

Asymptotic symmetry at null infinity is an infinite dimensional translational group (supertranslations) in four dimensions, not a four dimensional group. Then this implies that the angular momentum at null infinity has always ambiguities. Contrasted with this, it is shown that asymptotic symmetry at null infinity is the Poincare group in five dimensions. Then we can define the Bondi angular momentum at null infinity in a Poincare covariant way without any ambiguities.

There are remaining issues. In this paper we focused on the five-dimensional space-times. We would expect that our approach can be extended to higher dimensions than five. However, there is a critical point, that is, we had to introduce the concrete angular coordinate to solve the Einstein equation. On the other hand, one does not want to use the concrete angular coordinates when one is interested in higher dimensions. We have to resolve this troublesome issue. It is also interesting to study the asymptotic structure at null infinity in even dimensions using the Bondi coordinate because the finiteness has not been shown in even dimensions. They are left for future works.

\section{ACKNOWLEDGMENTS}

K.T. is supported by JSPS Grant-Aid for Scientific Research (Grant No. 21-2105). N.T. is supported by the DOE Grant DE-FG03-91ER40674, and thank Takahiro Tanaka for his grateful aid. T.S. is partially supported by Grant-Aid for Scientific Research from Ministry of Education, Science, Sports and Culture of Japan (Grant Nos. 21244033, 21111006, 20540258, and 19GS0219). This work is also supported by the Grant- in-Aid for the Global COE Program "The Next Generation of Physics, Spun from Universality and Emergence" from the Ministry of Education, Culture, Sports, Science and Technology (MEXT) of Japan.

\section{APPENDIX: ANGULAR MOMENTUM AT NULL INFINITY IN FOUR DIMENSIONS}

In this Appendix, we discuss the angular momentum at null infinity in four dimensions using the Bondi coordinates. This will be useful for the comparison with five-dimensional cases. We could not find old studies on angular momentum based on the Bondi coordinate. Here we will show that the angular momentum has always supertranslational ambiguities, which is represented by supermomentum.

\section{Bondi coordinate and Einstein equations in four dimensions}

In this section, we introduce the Bondi coordinates and solve the Einstein equations in four dimensions. ${ }^{5,6}$ In the Bondi coordinates $(u, r, \theta, \phi)$ the metric can be written as

$$
d s^{2}=-\frac{V e^{B}}{r} d u^{2}-2 e^{B} d u d r+r^{2} h_{A B}\left(d x^{A}+U^{A} d u\right)\left(d x^{B}+U^{B} d u\right),
$$

where

$$
h_{A B}=\left(\begin{array}{cc}
e^{C} & \sin \theta \sinh D \\
\sin \theta \sinh D & e^{E} \sin ^{2} \theta
\end{array}\right) .
$$


There is a gauge condition such that $\operatorname{det} h_{A B}=\sin ^{2} \theta$, and then $e^{E}$ can be written by $C$ and $D$ as

$$
e^{E}=e^{-C}\left(1+\sinh ^{2} D\right) \text {. }
$$

As in five dimensions, the functions $C$ and $D$ represent the degree of freedom of gravitational field. Given the function $C$ and $D$ on $u=$ const. hypersurfaces, the other metric functions $B, U^{A}$, and $V$ are determined by the Einstein equations. To solve the Einstein equations near null infinity, we expand the function $C$ and $D$ as

$$
\begin{aligned}
& C\left(u, r, x^{A}\right)=\frac{C_{1}\left(u, x^{A}\right)}{r}+\frac{C_{2}\left(u, x^{A}\right)}{r^{2}}+O\left(r^{-3}\right), \\
& D\left(u, r, x^{A}\right)=\frac{D_{1}\left(u, x^{A}\right)}{r}+\frac{D_{2}\left(u, x^{A}\right)}{r^{2}}+O\left(r^{-3}\right) .
\end{aligned}
$$

Then, using the Einstein equations $R_{a b}=0$, the metric functions $V, B$, and $U^{A}$ can be written by $C$ and $D$. From $R_{r r}=0$,

$$
\begin{gathered}
B\left(u, r, x^{A}\right)=\frac{B_{1}\left(u, x^{A}\right)}{r^{2}}+O\left(r^{-3}\right), \\
B_{1}\left(u, x^{A}\right)=-\frac{1}{8}\left(C_{1}^{2}+D_{1}^{2}\right) .
\end{gathered}
$$

From $R_{r A}=0$,

$$
\begin{gathered}
U^{A}\left(u, x^{A}\right)=\frac{U_{1}^{A}\left(u, x^{A}\right)}{r^{2}}+\frac{U_{2}^{A}\left(u, x^{A}\right)}{r^{3}}+O\left(r^{-4}\right), \\
U_{1}^{\theta}=\frac{1}{2 \sin ^{2} \theta}\left(\frac{\partial}{\partial \theta}\left(\sin ^{2} \theta C_{1}\right)+\frac{\partial}{\partial \phi}\left(\sin \theta D_{1}\right)\right) \\
\sin ^{2} \theta U_{1}^{\phi}=\frac{1}{2}\left(\frac{1}{\sin \theta} \frac{\partial}{\partial \theta}\left(\sin ^{2} \theta D_{1}\right)-\frac{\partial}{\partial \phi} C_{1}\right)
\end{gathered}
$$

From $h^{A B} R_{A B}=0$,

$$
\frac{V}{r}=1-\frac{m\left(u, x^{A}\right)}{r}+O\left(r^{-2}\right)
$$

Since the functions $U_{2}^{A}\left(u, x^{A}\right)$ and $m\left(u, x^{A}\right)$ are the integration constants in the $r$-integration, they are free functions of $\left(u, x^{A}\right)$.

\section{Bondi mass and angular momentum}

Now, $g_{u u}$ is expanded as

$$
g_{u u}=-1+\frac{m\left(u, x^{A}\right)}{r}+O\left(r^{-2}\right),
$$

and then we define the Bondi mass $M_{\text {Bondi }}$ and the Bondi momentum $P_{\text {Bondi }}^{i}$ in four dimensions as ${ }^{26}$

$$
\begin{gathered}
M_{\text {Bondi }}(u)=\frac{1}{8 \pi} \int_{S^{2}} m\left(u, x^{A}\right) d \Omega, \\
P_{\text {Bondi }}^{i}(u)=\frac{1}{8 \pi} \int_{S^{2}} m\left(u, x^{A}\right) \hat{x}^{i} d \Omega,
\end{gathered}
$$

where $d \Omega=\sin \theta d \theta d \phi$ and $\hat{x}^{i}=(\hat{x}, \hat{y}, \hat{z})=(\sin \theta \cos \phi, \sin \theta \sin \phi, \cos \theta)$. 
From the Einstein equation $R_{u u}=0$, we can obtain the Bondi mass loss law by gravitational waves as

$$
\begin{aligned}
\frac{d}{d u} M_{\text {Bondi }}(u) & =-\frac{1}{16 \pi} \int_{S^{2}}\left[\left(\frac{\partial C_{1}}{\partial u}\right)^{2}+\left(\frac{\partial D_{1}}{\partial u}\right)^{2}-\frac{1}{\sin \theta} \frac{\partial}{\partial \theta}\left(\sin \theta \frac{\partial U_{1}^{\theta}}{\partial u}\right)-\frac{\partial^{2}}{\partial \phi \partial u} U_{1}^{\phi}\right] d \Omega . \\
& <0 .
\end{aligned}
$$

Thus, the Bondi mass in four dimensions is always decreased by gravitational waves.

$g_{u \phi}$ are expanded as

$$
g_{u \phi}=\sin ^{2} \theta U_{1}^{\phi}+\frac{\sin \theta D_{1} U_{1}^{\theta}-\sin ^{2} \theta C_{1} U_{1}^{\phi}+\sin ^{2} \theta U_{2}^{\phi}}{r}+O\left(r^{-2}\right),
$$

and then we define the Bondi angular momentum in four dimensions as

$$
J_{\text {Bondi }}(u)=-\frac{3}{16 \pi} \int_{S^{2}}\left(\sin \theta D_{1} U_{1}^{\theta}-\sin ^{2} \theta C_{1} U_{1}^{\phi}+\sin ^{2} \theta U_{2}^{\phi}\right) d \Omega .
$$

From the Einstein equation $R_{u \phi}=0$, we can see that the evolution equation for the Bondi angular momentum becomes

$$
\frac{d}{d u} J_{\text {Bondi }}=-\frac{3}{16 \pi} \int_{S^{2}}\left[\left(\frac{\partial j}{\partial u}\right)_{\text {radiation }}+\left(\frac{\partial j}{\partial u}\right)_{\text {total derivative }}\right] d \Omega,
$$

where $(\partial j / \partial u)_{\text {radiation }}$ is the radiation part given by

$$
\begin{aligned}
\left(\frac{\partial j}{\partial u}\right)_{\text {radiation }}= & -\frac{1}{6 \sin \theta} \frac{\partial C_{1}}{\partial u} \frac{\partial}{\partial \theta}\left(\sin ^{2} \theta D_{1}\right)+\frac{1}{6 \sin \theta} \frac{\partial D_{1}}{\partial u} \frac{\partial}{\partial \theta}\left(\sin ^{2} \theta C_{1}\right)-\frac{1}{3} \frac{\partial D_{1}}{\partial u} \frac{\partial D_{1}}{\partial \phi}-\frac{1}{3} \frac{\partial C_{1}}{\partial u} \frac{\partial C_{1}}{\partial \phi} \\
& -\frac{\sin \theta}{6} \frac{\partial C_{1}}{\partial u} \frac{\partial D_{1}}{\partial \theta}+\frac{\sin \theta}{6} \frac{\partial D_{1}}{\partial u} \frac{\partial C_{1}}{\partial \theta},
\end{aligned}
$$

and $(\partial j / \partial u)_{\text {total derivative }}$ is total derivative given by

$$
\begin{aligned}
\left(\frac{\partial j}{\partial u}\right)_{\text {total derivative }}= & \frac{1}{3} \frac{\partial}{\partial \phi} m-\frac{\cot \theta}{3} \frac{\partial}{\partial \phi} U_{1}^{\theta}+\frac{1}{3} \frac{\partial^{2}}{\partial \phi \partial \theta} U_{1}^{\theta}+\frac{2 \sin ^{2} \theta}{3} U_{1}^{\phi} \\
& -\frac{1}{3 \sin \theta} \frac{\partial}{\partial \theta}\left(\sin ^{3} \theta \frac{\partial}{\partial \theta} U_{1}^{\phi}\right)-\frac{1}{3} \frac{\partial^{2}}{\partial u \partial \phi} C_{2} \\
& +\frac{1}{3 \sin \theta} \frac{\partial}{\partial \theta}\left(\sin ^{2} \theta \frac{\partial D_{2}}{\partial u}\right)-\frac{1}{3} \frac{\partial^{2}}{\partial u \partial \phi} B_{1} \\
& +\frac{1}{2} \frac{\partial}{\partial \phi}\left(D_{1} \frac{\partial}{\partial u} D_{1}\right)+\frac{1}{6} \frac{\partial}{\partial \phi}\left(C_{1} \frac{\partial}{\partial u} C_{1}\right) \\
& -\frac{1}{2 \sin \theta} \frac{\partial}{\partial \theta}\left(\sin ^{2} \theta C_{1} \frac{\partial}{\partial u} D_{1}\right)+\frac{1}{2 \sin \theta} \frac{\partial}{\partial \theta}\left(\sin ^{2} \theta D_{1} \frac{\partial}{\partial u} C_{1}\right) .
\end{aligned}
$$

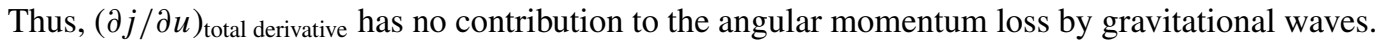

\section{Asymptotic symmetry and supermomentum}

Asymptotic symmetry is transformation group which satisfy the conditions given by

$$
\begin{gathered}
\delta g_{r r}=0, \delta g_{r A}=0, g^{A B} \delta g_{A B}=0, \\
\delta g_{u u}=O\left(r^{-1}\right), \delta g_{u A}=O(1), \delta g_{A B}=O(r),
\end{gathered}
$$

where $\delta g_{a b}=\nabla_{a} \xi_{b}+\nabla_{b} \xi_{a}$ is the infinitesimal transformation by $\xi$. These are required to keep the asymptotic behavior of the metric to be unchanged. 
From the conditions (A21), which come from the definition of the Bondi coordinate, we can check that the components of $\xi^{a}$ should have the following form:

$$
\begin{gathered}
\xi_{r}=f\left(u, x^{A}\right) e^{B}, \\
\xi_{B} g^{A B}=f^{A}\left(u, x^{A}\right)-f\left(u, x^{A}\right) U^{A}+\int_{r}^{\infty} d r^{\prime} e^{B} \frac{\partial f}{\partial x^{B}} g^{A B}, \\
\xi_{u}=-\frac{r e^{B}}{2}\left(-\frac{\partial \xi_{A}}{\partial x^{B}}+\xi_{C} \Gamma_{A B}^{C}+\xi_{r} \Gamma_{A B}^{r}\right) g^{A B} .
\end{gathered}
$$

From the conditions (A22), which are required from the behavior of the gravitational fields near null infinity, $f^{A}$ should satisfy

$$
\begin{aligned}
\frac{\partial}{\partial u} f^{A} & =0 \\
\mathcal{D}_{A} f_{B}+\mathcal{D}_{B} f_{A} & =-2 \frac{\partial f}{\partial u} h_{A B}^{(0)},
\end{aligned}
$$

where $\mathcal{D}_{A}$ is the covariant derivative with $h_{A B}^{(0)}$ given by

$$
h_{A B}^{(0)}=\left(\begin{array}{cc}
1 & 0 \\
0 & \sin ^{2} \theta
\end{array}\right) \text {. }
$$

$f^{A}$ generate the conformal transformation group on $S^{2}$ and such group is isomorphic to the Lorentz group in four dimensions. Then $f^{A}$ stands for the generator of the Lorentz transformations. Contrasted with in five dimensions, there are no further conditions on $f$ in four dimensions. Thus Eq. (A27) tells us that $f$ can be written as $f=-(u / 2) D^{A} f_{A}+\alpha\left(x^{A}\right) . \alpha\left(x^{A}\right)$ is arbitrary function on $S^{2}$ which is called supertranslations.

Now, we consider the transformation of the Bondi mass $M_{\text {Bondi }}$ by supertranslations $f=\alpha\left(x^{A}\right)$. $M_{\text {Bondi }}$ is transformed as

$$
\begin{gathered}
M_{\text {Bondi }} \rightarrow M_{\text {Bondi }}+\frac{1}{8 \pi} \int_{S^{2}}\left[-f \frac{\partial m}{\partial u}-\frac{\partial f}{\partial \theta} \frac{\partial U_{1}^{\theta}}{\partial u}-\frac{\partial f}{\partial \phi} \frac{\partial U_{1}^{\phi}}{\partial u}\right] \\
=M_{\text {Bondi }}+\frac{1}{16 \pi} \int_{S^{2}} f\left[\left(\frac{\partial C_{1}}{\partial u}\right)^{2}+\left(\frac{\partial D_{1}}{\partial u}\right)^{2}\right] .
\end{gathered}
$$

This is the Poincare transformation under the presence of gravitational waves. In four dimensions we cannot show the Poincare covariance of the Bondi momentum because $\partial U_{1}^{A} / \partial u \neq 0$ in the presence of gravitational waves. This means that the Bondi momentum has supertranslational ambiguities in four dimensions.

Next, we consider the transformations of the Bondi angular momentum by supertranslations. Angular momentum $J_{\text {Bondi }}$ is transformed as

$$
\begin{aligned}
J_{\text {Bondi }} \rightarrow & J_{\text {Bondi }}-\frac{3}{16 \pi} \int_{S^{2}}\left[-f \frac{\partial}{\partial u}\left(\sin \theta D_{1} U_{1}^{\theta}-\sin ^{2} \theta C_{1} U_{1}^{\phi}+\sin ^{2} \theta U_{2}^{\phi}\right)\right. \\
& -m \frac{\partial f}{\partial \phi}+\frac{1}{2} U_{1}^{\theta} \frac{\partial^{2}}{\partial \theta \partial \phi} f-\frac{1}{2} \frac{\partial f}{\partial \theta} \frac{\partial}{\partial \phi} U_{1}^{\theta} \\
& +\frac{1}{2} \frac{\partial f}{\partial \phi} \frac{\partial}{\partial \phi} U_{1}^{\phi}+\sin ^{2} \theta \frac{\partial f}{\partial \theta} \frac{\partial}{\partial \theta} U_{1}^{\phi}+\frac{1}{2} U_{1}^{\phi} \frac{\partial^{2} f}{\partial \phi^{2}}+2 \sin \theta \cos \theta U_{1}^{\phi} \frac{\partial f}{\partial \theta}+\frac{1}{3} \frac{\partial C_{2}}{\partial u} \frac{\partial f}{\partial \phi} \\
& -\frac{1}{3} \sin \theta \frac{\partial D_{2}}{\partial u} \frac{\partial f}{\partial \theta}+\frac{1}{3} \frac{\partial B_{1}}{\partial u} \frac{\partial f}{\partial \phi}-\frac{1}{2} \frac{\partial f}{\partial \phi} D_{1} \frac{\partial D_{1}}{\partial u}-\frac{1}{6} \frac{\partial f}{\partial \phi} C_{1} \frac{\partial C_{1}}{\partial u}+\frac{1}{2} \sin \theta \frac{\partial f}{\partial \theta} C_{1} \frac{\partial D_{1}}{\partial u}
\end{aligned}
$$




$$
\begin{aligned}
& \left.-\frac{1}{2} \sin \theta \frac{\partial f}{\partial \theta} D_{1} \frac{\partial C_{1}}{\partial u}+(\text { total derivative term })\right] d \Omega \\
= & J_{\text {Bondi }}-\frac{3}{16 \pi} \int_{S^{2}}\left[-f\left(\frac{\partial j}{\partial u}\right)_{\text {radiation }}+(\text { supermomentum })\right] d \Omega+\frac{1}{8 \pi} \int_{S^{2}} m \frac{\partial f}{\partial \phi} d \Omega,
\end{aligned}
$$

where supermomentum term is

$$
\begin{aligned}
\text { (supermomentum) }= & \frac{2}{3} f\left(2 \cot \theta \frac{\partial}{\partial \phi} U_{1}^{\theta}+\frac{\partial^{2}}{\partial \theta \partial \phi} U_{1}^{\theta}\right) \\
& -\frac{2}{3} f\left(6 \cos ^{2} \theta U_{1}^{\phi}-2 \sin ^{2} \theta U_{1}^{\phi}+6 \sin \theta \cos \theta \frac{\partial}{\partial \theta} U_{1}^{\phi}+\sin ^{2} \theta \frac{\partial^{2}}{\partial \theta^{2}} U_{1}^{\phi}\right) .
\end{aligned}
$$

If we take $f$ as $l=1$ mode of scalar harmonics on $S^{2}$, the supermomentum terms become total derivative form, and then we can obtain the Poincare covariance of the angular momentum $M_{\hat{x} \hat{y}} \rightarrow$ $M_{\hat{x} \hat{y}}+2 P_{[\hat{x}} \omega_{\hat{y}]}-f(d j / d u)_{\text {radiation }}$. However, for $l>1$ mode, the supermomentum terms do not become total derivative form. This means that we cannot obtain the Poincare covariance of angular momentum. Under the presence of gravitational waves, we cannot restrict $f$ to $l=1$ mode. In general, therefore, the angular momentum has supertranslational ambiguity in four dimensions.

${ }^{1}$ A. Ashtekar and R. O. Hansen, J. Math. Phys. 19, 1542 (1978).

${ }^{2}$ R. P. Geroch, "Asymptotic structure of space-time," in Asymptotic Structure of Space-Time, edited by T. P. Esposito and

L. Witten (Plenum, New York, 1977).

${ }^{3}$ R. Geroch, J. Math. Phys. 13, 956 (1972).

${ }^{4}$ K. Tanabe, N. Tanahashi, and T. Shiromizu, J. Math. Phys. 50, 072502 (2009).

${ }^{5}$ H. Bondi, M. G. J. van der Burg, and A. W. K. Metzner, Proc. R. Soc. London, Ser. A 269, 21 (1962).

${ }^{6}$ R. K. Sachs, Proc. R. Soc. London, Ser. A 270, 103 (1962).

${ }^{7}$ R. K. Sachs, Phys. Rev. 128, 2851 (1962).

${ }^{8}$ R. Penrose, Phys. Rev. Lett. 10, 66 (1963).

${ }^{9}$ E. T. Newman and R. Penrose, J. Math. Phys. 7, 863 (1966).

${ }^{10}$ R. Penrose, Proc. R. Soc. London, Ser. A 284, 159 (1965).

${ }^{11}$ R. P. Geroch and G. T. Horowitz, Phys. Rev. Lett. 40, 203 (1978).

${ }^{12}$ R. P. Geroch and B. C. Xanthopoulos, J. Math. Phys. 19, 714 (1978).

${ }^{13}$ S. Hollands and A. Ishibashi, J. Math. Phys. 46, 022503 (2005).

${ }^{14}$ S. Hollands and A. Ishibashi, e-print arXiv:hep-th/0311178.

${ }^{15}$ A. Ishibashi, Class. Quantum Grav. 25, 165004 (2008).

${ }^{16}$ K. Tanabe, N. Tanahashi, and T. Shiromizu, J. Math. Phys. 51, 062502 (2010).

${ }^{17}$ S. Hollands and R. M. Wald, Class. Quantum Grav. 21, 5139 (2004).

${ }^{18}$ L. A. Tamburino and J. H. Winicour, Phys. Rev. 150, 1039 (1966).

${ }^{19}$ J. H. Winicour, J. Math. Phys. 9, 861 (1968)

${ }^{20}$ C. R. Prior, Proc. R. Soc. London, Ser. A 354, 379 (1977).

${ }^{21}$ M. Streubel, Gen. Relativ. Gravit. 9, 551 (1978).

22 J. H. Winicour, "Angular momentum in general relativity," in General Relativity and Gravitation, edited by A. Held (Plenum, New York, 1980), Vol. 2.

${ }^{23}$ R. P. Geroch and J. Winicour, J. Math. Phys. 22, 803 (1981).

${ }^{24}$ T. Dray, and M. Streubel, Class. Quantum Grav. 1, 15 (1984).

${ }^{25}$ T. Harmark, Phys. Rev. D 70, 124002 (2004).

${ }^{26}$ The coefficients of the definitions are determined so that these quantities are coincide to ADM quantities at spatial infinity. 